\title{
ON THE LOCAL STABILITY OF DIFFERENTIAL FORMS $\left({ }^{1}\right)$
}

BY

\author{
MARTIN GOLUBITSKY AND DAVID TISCHLER
}

\begin{abstract}
In this paper we determine which germs of differential sforms on an $n$-manifold are stable (in the sense of Martinet). We show that when $s \neq 1$ or when $s=1$ and $n<4$ Martinet had found almost all of the possible examples. The most interesting result states that for certain generic singularities of 1-forms on 4-manifolds an infinite dimensional moduli space occurs in the classification of the 1-forms with this given singularity type up to equivalence by pull-back via a diffeomorphism.
\end{abstract}

In [4], Martinet proposed the following definition for stability of germs of differential s-forms on an $n$-manifold $M$. (Note. Throughout this paper all objects will be assumed to be $C^{\infty}$ differentiable.)

Definition 0.1. Let $w$ and $w^{\prime}$ be germs of s-forms on $M$ at $p$ and $p^{\prime}$ respectively. Then $(w, p)$ and $\left(w^{\prime}, p^{\prime}\right)$ are equivalent if there exists a germ of a diffeomorphism $f:(M, p) \rightarrow\left(M, p^{\prime}\right)$ such that $f^{*} w^{\prime}=w$ as germs near $p$.

Definition 0.2. Let $w$ be an s-form on $M$ at $p$. Then $w$ is stable at $p$ if for any nbhd $U$ of $p$ there is a nbhd $V$ of $w$ (in the $C^{\infty}$ topology on s-forms) such that if $w^{\prime}$ is in $V$, then there is a point $p^{\prime}$ in $U$ such that $(w, p)$ and $\left(w^{\prime}, p^{\prime}\right)$ are equivalent germs.

Clearly this definition depends only on the germ of $w$ at $p$.

Using this definition, Martinet constructs several examples of stable germs of forms. We shall show, using results of Martinet and Hsiung [3], that when $s \neq 1$ or when $s=1$ and $n \leqslant 4$ the examples of Martinet are essentially the only examples of stable germs. The only new additions are in the case of $(n-1)$-forms. We conjecture that in the remaining case when $s=1$ and $\operatorname{dim} M>4$ Martinet's examples are the only examples of locally stable forms. We also show that there are no stable germs of $s$-forms for $2 \leqslant s \leqslant n-2$ (Theorem 3.1).

The most satisfactory case in the determination of stable germs of s-forms occurs when $s=n-1$ (Theorem 2.5). Here there is a reasonable theory which classifies these stable forms according to singularity type. This classification is

Received by the editors July 3, 1975.

AMS (MOS) subject classifications (1970). Primary 58A10; Secondary 57D45, $58 \mathrm{C25}$.

( ${ }^{1}$ )This research was supported by NSF Contract GP 43524 and by the Research Foundation of CUNY, RF No. 11102 and RF No. 11105. 
given by the order of contact at $p$ of the line field $\operatorname{Ker} w$ with the hypersurface $\{d w=0\}$ where $w$ is the germ of the $(n-1)$-form at $p$.

Clearly the classification of stable germs of differential forms is but a small part of the determination of all the equivalence classes for these forms. This larger classification problem makes its appearance when we try to show that a given form is not stable. Usually we have had to exhibit small perturbations of the original form which belong to different equivalence classes. In this regard, the most interesting examples occur in the case of 1-forms on 4-manifolds. Here we find that the various singularity types of germs of 1 -forms (as described by Martinet) give rise to a rich and rigid geometric structure. What we show is that, except in the simplest cases, these singualrity types do not come close to describing the equivalence classes of germs of 1 -forms. In fact, for at least two distinct types of singularities, an infinite dimensional moduli space appears in the classification of equivalence classes of forms with the same singularity type.

See Proposition 4.7 and the proof of Theorem 4.11.

Our order is as follows: we start with 0 - and $n$-forms, do $(n-1)$-forms, then show that there are no locally stable $s$-forms when $2 \leqslant s \leqslant n-2$, and end with 1-forms.

First some notation. Let $\Lambda_{p}^{s}=\Lambda_{p}^{s}(M)$ denote the vector space of exterior $s$-forms on $T_{p} M$ where $T_{p} M$ denotes the tangent space of $M$ at $p$. Let $D_{p}^{s}=$ $D_{p}^{s}(M)$ denote the germs of differential s-forms on $M$ at $p$, and let $d: D^{s}(M) \rightarrow$ $D^{s+1}(M)$ denote exterior differentiation. Let $w$ be an $(s+1)$-form and $V$ a vector field on $M$. Then $V\lrcorner w$ denotes the $s$-form on $M$ obtained by contracting $\boldsymbol{w}$ by $V$.

DEFINITION 0.3. An invariant of the equivalence class of s-forms is an assignment of a number, function, germ, etc. defined on some open set of germs of $s$-forms which is identical for any two equivalent germs.

1. $n$-forms and 0 -forms. Let $w$ be an $n$-form on $M$. Martinet [4, p. 144] and Hsiung [3, Theorem 2.2] show that $w$ is stable at $p$ iff either

(a) $w_{p} \neq 0$ or

(b) $w_{p}=0$ and $(d h)_{p} \neq 0$ where $w=h v, h$ is the germ of a function mapping $(M, p) \rightarrow(\mathbf{R}, 0)$ and $v$ is the germ of a volume form at $p$.

Furthermore there exist coordinates $x_{1}, \ldots, x_{n}$ on $M$ at $p$ such that in case

(a) $w=d x_{1} \wedge \cdots \wedge d x_{n}$ and in case

(i) $w=x_{1} d x_{1} \wedge \cdots \wedge d x_{n}$.

A 0 -form $w$ is just a function. Clearly if $d w(p)=0$ then generically $w$ is a Morse function, so the critical point $p$ is isolated. For such forms, the critical value $w(p)$ is an invariant of the equivalence class of $w$ which is easily 
perturbed. So if $d w(p)=0, w$ is not stable at $p$. Conversely, if $d w(p) \neq 0$, then $w$ is stable at $p$.

2. (n-1)-forms. Let $w$ be the germ of an $(n-1)$-form. The following argument is mostly due to Hsiung [3, p. 8, Theorem 2.3].

Lemma 2.1. Suppose that $w(p)=0$, then $w$ is not stable at $p$.

Proof. First note that if $w$ were stable at $p$, then the zero of $w$ at $p$ would have to be transverse to the 0 -section in $\Lambda^{n-1}(M)$ and thus be isolated. So if $w^{\prime}$ is a small perturbation of $w$ at $p$, then $w^{\prime}$ would also have an isolated zero at some point $p^{\prime}$ near $p$. Hence if $f$ were a diffeomorphism such that $w=f^{*} w^{\prime}$ as germs at $p$, then $f(p)=p^{\prime}$.

Now suppose that $d w(p) \neq 0$. Then there exists a unique vector field $V$ on $M$ such that $w=V\lrcorner d w$. Clearly $V$ has an isolated zero on $M$ at $p$. Defining $V^{\prime}$ similarly for $w^{\prime}$, we see that if $f^{*} w^{\prime}=w$ at $p$, then $f_{*} V=V^{\prime}$. So the eigenvalues of the linear part of $V$ at $p$ are invariants of the equivalence class of $w$ at $p$. We claim that a small perturbation of $w$ will change these eigenvalues, so that $w$ is not stable at $p$. To see this, let $w^{\prime}=w+d \sigma$ where $\sigma$ is the germ of an $(n-2)$-form on $M$ at $p$ and $d \sigma(p)=0$. Then $V^{\prime}=V+W$ where $\left.W\right\lrcorner d w=d \sigma$. Since $d w$ is a volume form and $d \sigma$ is closed, the only restriction that we put on the perturbation $W$ is that it be a volume preserving vector field (relative to $d w$ ). This means that the trace of the linear part of $W$ at $p$ is 0 . Clearly there is a $W$ so that the eigenvalues of $V^{\prime}$ are different from those of $V$.

Next note that it is generically impossible for both $w$ and $d w$ to be 0 at $p$, which finishes the lemma. But in certain applications of this lemma we will have the situation where $d w(p)$ is constrained to be zero. Even so the lemma is true. Let $\Omega$ be any volume form on $M$. Then as above there is a unique vector field $V$ on $M$ at $p$ such that $w=V\lrcorner \Omega$. Had we chosen another volume form $\Omega^{\prime}$ then the corresponding vector field $V^{\prime}$ would be a nonzero function multiple of $V$. In this case the eigenvalues of the linear part of $V$ are not invariants but the various ratios of these eigenvalues are invariants. Clearly the perturbations $W$ are numerous enough to change these ratios. So $w$ is not stable at $p$.

REMARK. We actually proved more than what was stated; namely if $w(p)=0$, then $w$ is not stable at $p$ under perturbations by closed forms.

Assuming that $w$ is stable at $p$, we have two cases, $d w(p) \neq 0$ or $d w(p)=$ 0 . The first case is Martinet [4, p. 146].

LEMMA 2.2. If $w(p) \neq 0$ and $d w(p) \neq 0$, then there exist coordinates $x_{1}, \ldots, x_{n}$ on $M$ at $p$ such that $w=\left(1+x_{1}\right) d x_{2} \wedge \cdots \wedge d x_{n}$.

So we may assume that $d w(p)=0$ and $w(p) \neq 0$. Genericity implies that we may choose coordinates on $M$ at $p$ such that $d w=x_{1} d x_{1} \wedge \cdots \wedge d x_{n}$. See Martinet [4, p. 144]. 
Since $w(p) \neq 0$, there is a well-defined line field near $p$ given by $\operatorname{Ker} w$. Let $X=\{d w=0\}=\left\{x_{1}=0\right\}$. Again we have two cases: either $\operatorname{Ker} w \boldsymbol{X}$ at $p$ or $\operatorname{Ker} w(p) \subseteq T_{p} X$.

LEMMA 2.3 (MARTINET [4, p. 148]). Suppose that $w(p) \neq 0, d w(p)=0$, and $\operatorname{Ker} w \Phi$ at $p$. Then there exist coordinates $x_{1}, \ldots, x_{n}$ on $M$ at $p$ such that $w=\left(1 \pm x_{1}^{2} / 2\right) d x_{2} \wedge \cdots \wedge d x_{n}$.

Note. The sign of the $x_{1}^{2} / 2$ term is determined geometrically as follows: away from $X d w$ is a volume form so there is a well-defined vector field $V$ such that $V\lrcorner d w=w$. The sign of $x_{1}^{2} / 2$ is + if the one parameter group of $V$ moves away from $X$ and is - otherwise.

So now we may assume that $w(p) \neq 0, d w(p)=0$, and $\operatorname{Ker} w_{p} \subseteq T_{p} X$.

Let $J_{p}^{n+2}\left(\Lambda^{n-1}\right)$ be the manifold of $(n+2)$-jets of $(n-1)$-forms on $M$ at $p$. Let $W_{p}^{k}$ be the subset of $J_{p}^{n+2}\left(\Lambda^{n-1}\right)$ defined as follows: $w$ is in $W_{p}^{k}$ if

(1) $w(p) \neq 0$ and $d w(p)=0$.

(2) The zero of $d w$ is generic.

(3) $\operatorname{Ker} w(p) \subset T_{p} X$ where $X=\{d w=0\}$.

(4) The order of contact of the line field $\operatorname{Ker} w$ with $X$ is $k-2$.

LEMMA 2.4. $W_{p}^{k}$ is a submanifold of $J_{p}^{n+2}\left(\Lambda^{n-1}\right)$ of codimension $k-1$. (We assume that $k \leqslant n+2$.)

Proof. Choose coordinates $x_{1}, \ldots, x_{n}$ on $M$ at $p$ so that the line field Ker $w$ is generated by $\partial / \partial x_{n}$. Let $V$ be the vector field-defined off $X$ - such that $w=V\lrcorner d w$. Hence $V\lrcorner w=V\lrcorner(V\lrcorner d w)=0$ off $X$. So $w=\varphi d x_{1} \wedge$ $\ldots \wedge d x_{n-1}$ off $X$. By continuity $w=\varphi d x_{1} \wedge \ldots \wedge d x_{n-1}$ on a nbhd of $p$ where $\varphi:(M, p) \rightarrow \mathbf{R}$ is $C^{\infty}$. By a simple change of coordinates we may assume that $\varphi(0)=1$. With these coordinates $d w= \pm\left(\partial \varphi / \partial x_{n}\right) d x_{1} \wedge \cdots \wedge d x_{n}$. So $X$ is given by $\left\{\partial \varphi / \partial x_{n}=0\right\}$. In particular $\partial \varphi(0) / \partial x_{n}=0$. The genericity of the zero of $d w$ implies that $d\left(\partial \varphi / \partial x_{n}\right)_{0} \neq 0$. The fact that $\operatorname{Ker} w(p) \subset T_{p} X$ implies that $d\left(\partial \varphi / \partial x_{n}\right)_{0}\left(\partial / \partial x_{n}\right)=0=\partial^{2} \varphi(0) / \partial x_{n}^{2}$. The order of contact of the line field $\operatorname{Ker} d w$ with $X$ is given by the order of the zero of the function $d\left(\partial \varphi / \partial x_{n}\right)\left(\partial / \partial x_{n}\right)\left(0, \ldots, 0, x_{n}\right)$. To say that the order of contact is $k-2$ is equivalent to

$$
\partial \varphi(0) / \partial x_{n}=\cdots=\partial^{k-1} \varphi(0) / \partial x_{n}^{k-1}=0
$$

while $\partial^{k} \varphi(0) / \partial x_{n}^{k} \neq 0$. Thus $W_{p}^{k}$ is defined by $k-1$ independent equations and is a submanifold of codimension $k-1$ in $J_{p}^{n+2}\left(\Lambda^{n-1}\right)$.

Let $J^{n+2}\left(\Lambda^{n-1}\right)$ be the manifold of $(n+2)$-jets of $(n-1)$ forms on $M$ and $W^{k}=\bigcup_{p \in M} W_{p}^{k}$. Then $W^{k}$ is a submanifold of $J^{n+2}\left(\Lambda^{n-1}\right)$ and codim $W^{k}$ $=k-1$. If $w$ is the germ of a stable $(n-1)$-form at $p$ satisfying (1), (2), and 
(3), then $j^{n+2} w$ \$ $w^{k}$ at $p$. In particular there is a $k$ between 3 and $n+1$ satisfying (*) since codim $W^{n+2}$ is $n+1$ and transversality implies null intersection.

Let $w$ have the form $\varphi d x_{1} \wedge \cdots \wedge d x_{n-1}$ and satisfy $j^{n+2} w \aleph W^{k}$ at $p$ with $3 \leqslant k \leqslant n+1$. Think of $\varphi$ as an unfolding of the function $\varphi\left(0, \ldots, 0, x_{n}\right)$ parametrized by $x_{1}, \ldots, x_{n-1}$. Using the unfolding theorem (see, for example, $[5$, p. 375], [6], [7] $)$ we can find functions $\psi\left(x_{1}, \ldots, x_{n}\right)$ and $\lambda_{i}\left(x_{1}, \ldots, x_{n-1}\right)$ for $1 \leqslant i \leqslant n-1$ such that

(a) $\psi(0)=\lambda_{i}(0)=0$,

(b) $\mu\left(x_{1}, \ldots, x_{n}\right)= \pm \psi^{k}+\lambda_{k-2} \psi^{k-2}+\cdots+\lambda_{1} \psi+1$, and

(c) $\Psi=\left(\lambda_{1}, \ldots, \lambda_{n-1}, \psi\right)$ is a legitimate change of coordinates.

THEOREM 2.5. Suppose that $w$ is stable at $p, d w(p)=0$ and $\operatorname{Ker} w_{p} \subset$ $T_{p} X$ where $X=\{d w=0\}$. Then there exists a $k$ with $3 \leqslant k \leqslant n$ and coordinates $x_{1}, \ldots, x_{n}$ on $M$ at $p$ such that

$$
w=\left( \pm x_{n}^{k}+x_{k-2} x_{n}^{k-2}+\cdots+x_{1} x_{n}+1\right) d x_{1} \wedge \cdots \wedge d x_{n-1} \text {. }
$$

Moreover these germs are stable at 0.

Notes. (1) When $k$ is odd the $\pm x_{n}^{k}$ term can be assumed to be $x_{n}^{k}$.

(2) The sign of $x_{n}^{k}$ when $k$ is even is determined as in the note after Lemma 2.3.

(3) The statements of Lemmas 2.2 and 2.3 are given by $k=1$ and $k=2$ respectively. The proof of this theorem can be adapted to prove these lemmas as well.

(4) $k-2$ is just the order of contact of the line field Ker $w$ with the hypersurface $X$.

Proof. From the discussion above, compute $\Psi^{*} w=$ $\left( \pm \psi^{k}+\lambda_{k-2} \psi^{k-2}+\cdots+\lambda_{1} \psi+1\right) \cdot \mu\left(\lambda_{1}, \ldots, \lambda_{n-1}\right) d \lambda_{1} \wedge \cdots \wedge d \lambda_{n-1}$ where $\mu(0) \neq 0$ and $3 \leqslant k \leqslant n+1$.

If $k \leqslant n$, let $\bar{\lambda}_{n-1}=\int_{0}^{\lambda_{n-1}} \mu\left(\lambda_{1}, \ldots, \lambda_{n-2}, t\right) d t$. In the coordinates $\left(\lambda_{1}, \ldots, \lambda_{n-2}, \bar{\lambda}_{n-1}, \psi\right) w$ has the appropriate form. Since the conditions needed to obtain this normal form are all given by transversality statements, these germs are stable.

To complete the proof of this theorem we need to show that if $k=n+1$, then $w$ is not stable at $p$. By the above we may choose coordinates $x, y_{1}, \ldots$, $y_{n-1}$ so that

$$
w=\left( \pm x^{n+1}+y_{n-1} x^{n-1}+\cdots+y_{1} x+1\right) \mu(y) d y
$$

with $\mu(0) \neq 0$. Let $w_{a}=a w$ for some number $a$ near but $\neq 1$. We claim that at no point $q$ near 0 is the germ of $w_{a}$ at $q$ equivalent to the germ of $w$ at 0 . Note that for both $w$ and $w_{a}, 0$ is the only point where the order of contact 
of the line field $\operatorname{Ker} w$ with $X$ is $n-1$; so if $\sigma$ is a diffeomorphism satisfying $\sigma^{*} w=w_{a}$, then $\sigma(0)=0$. Let $\sigma(x, y)=(f(x, y), g(x, y))$ where $f:\left(\mathbf{R}^{n}, 0\right) \rightarrow$ $(\mathbf{R}, 0)$, and $g:\left(\mathbf{R}^{n}, 0\right) \rightarrow\left(\mathbf{R}^{n-1}, 0\right)$ be such a diffeomorphism. First note that $\sigma^{*} w=w_{a}$ implies that $\sigma_{*}(\operatorname{Ker} w)=\operatorname{Ker} w$. So $g=g(y)$. Hence $\sigma^{*} w=w_{a}$ is equivalent to

$$
\begin{aligned}
\left( \pm f^{n+1}+g_{n-1} f^{n-1}\right. & \left.+\cdots+g_{1} f+1\right) \mu(g) \operatorname{det}(d g) \\
& =a\left( \pm x^{n+1}+y_{n-1} x^{n-1}+\cdots+y_{1} x+1\right) \mu(y) .
\end{aligned}
$$

Evaluating (**) at 0 yields $\operatorname{det}(d g)_{0}=a$ while evaluating (**) at $y=0$ yields $f(x, 0)= \pm x$. Finally differentiating $(* *)$ with respect to $y_{j}$ and setting $y=0$ yields

$$
\pm \frac{\partial g_{n-1}}{\partial y_{j}}(0) x^{n-1} \pm \cdots \pm \frac{\partial g_{1}}{\partial y_{j}}(0) x=x^{j}
$$

Thus $\partial g_{i}(0) / \partial y_{j}= \pm \delta_{i j}$ and $\operatorname{det}(d g)_{0}= \pm 1$. Since $a \neq \pm 1$ we have a contradiction and no such diffeomorphism $\sigma$ exists. Thus when $k=n+1, w$ is not stable at 0 .

3. $s$-forms where $2 \leqslant s \leqslant n-2$. In this section we adapt Hsiung's theorem $[3$, p. 10 , Theorem 2.6$]$ that there are no infinitesimally stable $s$-forms $(2 \leqslant s \leqslant$ $n-2)$ to show

THEOREM 3.1. There are no stable germs of s-forms when $2 \leqslant s \leqslant n-2$.

Let $w$ be a stable $s$-form at $p$. Let $U$ be a coordinate nbhd of $p$ in $M$. In these coordinates we may assume that $U=\mathbf{R}^{n}$ and $p=0$. Since $w$ is stable at $p$ there is a nbhd $W$ of $w$ in $D^{s}(M)$ such that if $w^{\prime}$ is in $W$ then there is a germ of a diffeomorphism $f:(U, p) \rightarrow\left(U, p^{\prime}\right)$ with $\left(f^{*} w^{\prime}\right)_{p}=w_{p}$. Since $W$ is a nbdh of $w$ in the $C^{\infty}$ topology, it is also a nbhd of $w$ in the $C^{k}$ topology on $D^{s}(M)$ for all $k$ large enough.

Let $J^{k}\left(\Lambda^{s}\right)_{p}=$ the $k$-jets of $s$-forms at $p$. Consider the map $\Phi: W \times U \rightarrow$ $J^{k}\left(\Lambda^{s}\right)_{p}$ given by $\left(w^{\prime}, a\right) \mapsto j^{k}\left(a^{*} w^{\prime}\right)_{p}$ where $a$ in $U$ is viewed as a diffeomorphism of $U$ via translation in the coordinates of $U$ and $j^{k}()_{p}: D^{s} \rightarrow J^{k}\left(\Lambda^{s}\right)_{p}$ is the $k$-jet extension map on sections. Let $\Phi_{w^{\prime}}(a)=\Phi\left(w^{\prime}, a\right)$. Let $\Theta_{w}$ be the orbit through $w$ of the natural action of $\operatorname{Diff}_{p}(M)=$ group of germs of diffeomorphisms mapping $(M, p) \rightarrow(M, p)$. Let $\Theta_{w}^{k}=j^{k}\left(\Theta_{w}\right)_{p}$.

LEMMA 3.2. If $w$ is stable, then $\operatorname{Im} \Phi_{w^{\prime}} \cap \Theta_{w}^{k} \neq \varnothing$ for any $w^{\prime}$ in $W$, and any $k$.

Proof. Let $w^{\prime}$ be in $W$. Since $w$ is stable, there is a diffeomorphism $f:(M, p) \rightarrow\left(M, p^{\prime}\right)$ such that $\left(f^{*} w^{\prime}\right)=w$ as germs at $p$. Let $a=p^{\prime}$. Note in the coordinates on $M$ at $p, p=0$. Then $w=f^{*} w^{\prime}=(-a f)^{*} a^{*} w^{\prime}$ as germs at 
p. So $a^{*} w^{\prime}=\left(f^{-1} \circ a\right)^{*} w$ and $a^{*} w^{\prime}$ is in $\Theta_{w^{k}}^{k}$. Hence $\Phi_{w^{\prime}}(a)=j^{k}\left(a^{*} w^{\prime}\right)_{p}$ is in $\Theta_{w}^{k}$.

LEMMA 3.3. For $k$ large enough, codim $\Theta_{w}^{k}$ in $J^{k}\left(\Lambda^{s}\right)_{p}$ is greater than $n$ when $2 \leqslant s \leqslant n-2$. (We can assume $n \geqslant 4$.)

Proof. Let $\operatorname{Diff}_{p}^{k+1}(M)=$ group of invertible $(k+1)$-jets on $M$ at $p$. Clearly $\Theta_{w}^{k}$ is also given by the action of $\operatorname{Diff}_{p}^{k+1}(M)$ on $J^{k}\left(\Lambda^{s}\right)_{p}$. So

and

$$
\operatorname{dim} \Theta_{w}^{k} \leqslant \operatorname{dim} \operatorname{Diff}_{p}^{k+1}(M)=n\left(\begin{array}{c}
n+k+1 \\
n
\end{array}\right)
$$

$$
\begin{aligned}
\operatorname{codim} \Theta_{w}^{k} & =\operatorname{dim} J^{k}\left(\Lambda^{s}\right)_{p}-\operatorname{dim} \Theta_{w}^{k} \geqslant \operatorname{dim} J^{k}\left(\Lambda^{s}\right)_{p}-n\left(\begin{array}{c}
n+k+1 \\
n
\end{array}\right) \\
& =\left(\begin{array}{c}
n \\
s
\end{array}\right)\left(\begin{array}{c}
n+k \\
n
\end{array}\right)-n\left(\begin{array}{c}
n+k+1 \\
n
\end{array}\right) \\
& =\frac{(n+k) \cdots(n+1)}{k \cdots 1}\left[\left(\begin{array}{l}
n \\
s
\end{array}\right)-\frac{n(n+k+1)}{k+1}\right] \\
& \geqslant\left(1+\frac{n}{k}\right) \cdots\left(1+\frac{n}{1}\right)\left[\left(\begin{array}{l}
n \\
2
\end{array}\right)-n\left(1+\frac{n}{k+1}\right)\right]
\end{aligned}
$$

when $2 \leqslant s \leqslant n-2$. Hence codim $\Theta_{w}^{k} \geqslant(1+n / k)^{k}$ when $k$ is large and $n \geqslant$ 4. So as $k \rightarrow \infty$, codim $\Theta_{w}^{k} \geqslant e^{n}-1 \geqslant n$.

Proof of Theorfm 3.1. Consider $\Psi: J^{k}\left(\Lambda^{s}\right)_{p} \times U \rightarrow J^{k}\left(\Lambda^{s}\right)_{p}$ defined by $\Psi(v, a)=\Phi(w+v, a)$ where $\Phi$ is as above. Since $\Phi(w+v, 0)=j^{k}(w+v)_{p}$, $\Psi$ is a submersion near $(0,0)$ and $\Psi_{v}$ $\Theta_{w}^{k}$. Let $\Psi_{v}(a)=\Psi(v, a)$. By the fundamental lemma on transversality (see $[1$, p. 54]) there is a dense set of parameters $B \subset J^{k}\left(\Lambda^{s}\right)_{p}$ such that $\Psi_{v} \Theta_{w}^{k}$ when $v$ is in $B$. Choose $k$ large enough so that codim $\Theta_{w}^{k}>n$. Since $\Psi_{v}: U \rightarrow J^{k}\left(\Lambda^{s}\right)_{p}$ and $\operatorname{codim} \Theta_{w}^{k}>n$ we have that $\operatorname{Im} \Psi_{v} \cap \Theta_{w}=\varnothing$ for all $v$ in $B$. So for a set of germs $w+v$ arbitrarily close to $w, w+v$ at $p^{\prime}$ is not equivalent to $w$ at $p$ for any $p^{\prime}$ in $U$. Since for $v$ small enough $w+v$ is in $W$, we have that $w$ is not stable at $p$.

4. 1-forms. In this section we finish the classification of stable germs of 1 -forms on manifolds of dimension $\leqslant 4$. We show, in fact, that there are no examples except for the ones which Martinet computed.

$\S \S 1$ and 2 completed the classification of stable germs of 1 -forms on manifolds of dimensions 1 and 2 respectively.

First we assume that $n=\operatorname{dim} M=2 k+1$. Martinet's results are as follows: 
LEMMA 4.1. Let $w$ be the germ of a contact 1 -form on $M^{2 k+1}$ at $p$; that is, $w \wedge(d w)^{k}(p) \neq 0$. Then there exist coordinates $z, x_{1}, \ldots, x_{k}, y_{1}, \ldots, y_{k}$ at $p$ such that $w=d z+x_{1} d y_{1}+\cdots+x_{k} d y_{k}$.

The proof is classical; see, for example, $[4$, p. 152]

Let $w$ be the germ of a 1 -form on $M^{2 k+1}$ at $p$ satisfying $w \wedge(d w)^{k}(p)=$ 0 . If $w$ is stable, then we may assume that $S=\left\{q \in M \mid w \wedge(d w)^{k}(q)=0\right\}$ is a submanifold of codimension one. This is, of course, given by transversality. Let $l: S \hookrightarrow M$ be the inclusion map.

LEMMA 4.2. Let $w$ be the germ of a 1-form on $M^{2 k+1}$ at $p$ for which $w(p) \neq 0, w \wedge(d w)^{k}$ has a generic zero at $p$, and $l^{*}(d w)^{k}(p) \neq 0$. Then there exist coordinates $z, x_{1}, \ldots, x_{k}, y_{1}, \ldots, y_{k}$ on $M$ at $p$ such that

$$
w= \pm z d z+\left(1+x_{1}\right) d y_{1}+x_{2} d y_{2}+\cdots+x_{k} d y_{k} \text {. }
$$

Proof. See $[4$, p. 154$]$. Martinet actually assumes that $w(p) \neq 0$ without stating it.

Note. The sign of $z d z$ is given as follows: Let $V$ be the vector field defined off $S$ by $\left.(d w)^{k}=V\right\lrcorner w \wedge(d w)^{k}$. If the one parameter group of $V$ flows toward $S$, then the sign of $z d z$ is -; otherwise it is + .

Proposition 4.3. The only stable germs of 1 -forms on $M^{3}$ at $p$ are given by Lemmas 4.1 and 4.2 .

Proof. Let $w$ be the germ of a stable 1 -form on $M^{3}$ at $p$. This implies that $d w(p) \neq 0$. For if $w$ is stable at $p$, then $d w$ is stable amongst closed forms at $p$. Since $d w$ is a 2-form on a 3-manifold we can apply the Remark after Lemma 2.1. By eliminating the case considered in Lemma 4.1, we may assume that $w \wedge d w(p)=0$. As above $S=\{w \wedge d w=0\}$ is a two-dimensional submanifold of $M$. Let $l: S \hookrightarrow M$ be the inclusion map. Then $w$ stable at $p$ implies that $l^{*} w(p) \neq 0$; since $l^{*} w$ is a 1 -form on a 2 -manifold we can apply the same type of argument as in Lemma 2.1. Thus stability implies that $w(p) \neq 0$.

Eliminating the case considered in Lemma 4.2 yields the following situation; $w \wedge d w(p)=0, l^{*}(d w)(p)=0, l^{*} w(p) \neq 0$, and $d w(p) \neq 0$. Since $d w(p) \neq 0$ we can choose coordinates $x, y$, and $z$ on $M$ at $p$ such that $d w=d x d y$. Let $w=a d x+b d y$ $+c d z$. Since $w \wedge d w=c d x d y d z, S$ is defined by $c=0$. By a small perturbation of $w$, we can perturb the defining function for $S$, namely $c$, in any direction. Let $w^{\prime}=w+d H$. Then $w^{\prime} \wedge d w^{\prime}=(c+\partial H / \partial z) d x d y d z$. By choosing $H$ small we can perturb $c$ in any direction. View $c$ as an unfolding of the function $c(0,0, z)$ with parameters $x$ and $y$. Using transversality and the unfolding theorem $[5$, p. 375] $,[6],[7]$, there exist functions $\varphi(x, y, z), \lambda(x, y), \mu(x, y)$ with 
$\varphi(0)=0=\lambda(0)=\mu(0)$ such that $\Psi=(\lambda, \mu, \varphi)$ is a legitimate change of coordinates and either

(1) $c(x, y, z)=\varphi$,

(2) $c(x, y, z)= \pm \varphi^{2}-\lambda$, or

(3) $c(x, y, z)=\varphi^{3}-\lambda \varphi-\mu$.

This is similar to the situation for $(n-1)$-forms described in $\$ 2$. In the coordinates defined by $\Psi$ we have that $d w=f(x, y) d x d y$ for some function $f$ and $w=a d x+b d y+c d z$ where either

(i) $c(x, y, z)=z$,

(ii) $c(x, y, z)= \pm z^{2}-x$,

(iii) $c(x, y, z)=z^{3}-x z-y$.

In case (i), $S=\{z=0\}$ and $l^{*} d w=d x d y \neq 0$ on $S$. In both cases (ii) and (iii), $l^{*}(d w)(p)=0$. These cases are distinguished as follows: There is a welldefined line field given by $\operatorname{Ker} d w=(\partial / \partial z)$. For $l^{*} d w(p)$ to equal zero we must have that $\operatorname{Ker}(d w)_{p} \subset T_{p} S$. The order of contact of the line field $\operatorname{Ker}(d w)$ with $S$ distinguishes cases (ii) and (iii).

First we consider case (ii). From the above discussion, including the fact that $d w$ is independent of $z$, we have

$$
w=\left( \pm z^{2}+x\right) d z+(z+b(x, y)) d x+e(x, y) d y .
$$

In coordinates, $S=\left\{x= \pm z^{2}\right\}$. Let $T=\left\{l^{*} d w=0\right\}$. Then $T=\{x=z=0\}$. Let $\sigma$ be a diffeomorphism such that $\sigma^{*} w=a w$ for $a$ near but $\neq 1$. Then $\sigma_{*}(\operatorname{Ker} d w)=\operatorname{Ker} d w$ which implies that $\sigma_{*}(\partial / \partial z)=(\partial / \partial z)$. So if $\sigma=(f, g, h)$, then $\partial f / \partial z=\partial g / \partial z=0$. Since $\sigma(T)=T$ we have that $f(0, y)=0=h(0, y, 0)$. Hence $f_{y}(0)=0=h_{y}(0)$.

Matching the $d z$ terms in the equation $\sigma^{*} w=a w$ yields

$$
\left( \pm h^{2}+f\right) h_{z}=a\left( \pm z^{2}+x\right)
$$

Set $x=0$ in (1) to obtain $\pm h^{2} h_{z}= \pm a z^{2}$. So $h(0, y, z)=a^{1 / 3}$ and $h_{z}(0)=$ $a^{1 / 3}$. Next differentiate (1) by $x$ and evaluate at 0 to obtain $f_{x}(0)=a^{2 / 3}$.

Now suppose $e(0)=0$. Let $j: T \hookrightarrow R^{3}$ be the inclusion map. Then $j^{*} w=e(0, y) d y$. So the set $\left\{j^{*} w=0\right\}=e^{-1}(0) \cap T$. Generically this set is just a point. Since $\sigma$ must preserve the set $\left\{j^{*} w=0\right\}$ we have that $\sigma(0)=0$. Matching coefficients on the $d x$ term yields the equation

$$
(h+b(f, g)) f_{x}+e(f, g) g_{x}+\left(f \pm h^{2}\right) h_{x}=a(z+b(x, y)) .
$$

Evaluate $\left(2^{\prime}\right)$ at 0 to yield $b(0) f_{x}(0)=a b(0)$. Since $w(0) \neq 0$ and $e(0)=0$, we must have $b(0) \neq 0$. Thus $f_{x}(0)=a$ which contradicts our calculation above, since $a \neq 1$. So $w$ is not stable at $p$ and we may assume that $e(0) \neq 0$. 
Consider the change of coordinates $\bar{x}=x, \bar{y}=\int_{0}^{y} e(x, t) d t, \bar{z}=z$. This is a legitimate change of coordinates since $e(0) \neq 0$. In these coordinates (dropping the bars) $w$ has the form

$$
w=(z+b(x, y)) d x+d y+\left(x \pm z^{2}\right) d z .
$$

Equation $\left(2^{\prime}\right)$ now has the form

$$
(h+b(f, g)) f_{x}+g_{x}+\left( \pm h^{2}+f\right) h_{x}=a(z+b(x, y)) .
$$

Matching coefficients on $d y$ yields

$$
(h+b(f, g)) f_{y}+g_{y}+\left(f \pm h^{2}\right) h_{y}=a .
$$

The equation $\sigma^{*} d w=a d w$ yields

$$
b_{y}(f, g) \operatorname{det}\left(d(f, g)_{(x, y)}\right)=b_{y}(x, y) .
$$

Evaluate (3) at 0 to obtain $g_{y}(0)=a$. Thus $\operatorname{det}\left(d(f, g)_{0}\right)=a^{5 / 3}$ since $f_{x}(0)=$ $a^{2 / 3}$ and $f_{y}(0)=0$. All that we know about $\sigma(0)$ is that it must be in $T$. So let $g_{0}=g(0)$. Evaluate (4) at 0 to see that $b_{y}\left(0, g_{0}\right)=a^{-5 / 3} b_{y}(0)$. Next differentiate (2) by $y$ and evaluate at 0 to obtain

$$
b\left(0, g_{0}\right) f_{x y}(0)+g_{x y}(0)=b_{y}(0)[a-1] .
$$

Finally differentiate (3) by $x$ and evaluate at 0 to obtain

$$
b\left(0, g_{0}\right) f_{y x}(0)+g_{y x}(0)=0 .
$$

Note that $d w(0) \neq 0$ implies that $b_{y}(0) \neq 0$. So $a=1$. This is a contradiction so $w$ is not stable at 0 .

Finally we consider case (iii). We have that

$$
w=\left(z^{3}+x z+y\right) d z+\left(z^{2} / 2+b(x, y)\right) d x+(z+e(x, y)) d y .
$$

Again, suppose that $\sigma$ is a diffeomorphism such that $\sigma^{*} w=a w$ for $a$ near but $\neq$ 1. Note that for both $w$ and $a w, 0$ is the unique point where Ker $d w=\operatorname{Ker} a d w$ $=(\partial / \partial z)$ has contact of order 1 with $S$, so $\sigma(0)=0$. Let $\sigma=(f, g, h)$. Since $\sigma_{*}(\operatorname{Ker} d w)=\operatorname{Ker}(d w)$ we have that $\partial f / \partial z=\partial g / \partial z=0$. So $\sigma^{*} w=a w$ yields

and

$$
\left(h^{3}+f h+g\right) \partial h / \partial z=a\left(z^{3}+x z+y\right)
$$

$$
\begin{aligned}
\left(h^{3}+f h+g\right) \frac{\partial h}{\partial y}+\left(\frac{h^{2}}{2}+b(f, g)\right) \frac{\partial f}{\partial y}+(h+e(f, g)) \frac{\partial g}{\partial y} & =a(z+e(x, y)),
\end{aligned}
$$

$$
\begin{aligned}
\left(h^{3}+f h+g\right) \frac{\partial h}{\partial x}+\left(\frac{h^{2}}{2}+b(f, g)\right) \frac{\partial f}{\partial x}+(h+e(f, g)) \frac{\partial g}{\partial x} \\
=a\left(\frac{z^{2}}{2}+b(x, y)\right) .
\end{aligned}
$$


Evaluate (5) at $x=y=0$ to yield $h(0,0, z)= \pm a^{1 / 4} z$. Evaluate (6) and (7) at 0 to yield

$$
b(0) f_{y}(0)+e(0) g_{y}(0)=a e(0)
$$

and

$$
b(0) f_{x}(0)+e(0) g_{x}(0)=a b(0)
$$

Differentiate (5) with respect to $y$ and evaluate at 0 to obtain $\partial g(0) / \partial y=a^{3 / 4}$. Differentiate (5) with respect to $x$ and evaluate at $x=y=0$. Now equate the coefficients of the linear terms to obtain $\partial g(0) / \partial x=0$ and $\partial f(0) / \partial x=a^{1 / 2}$.

Substitute in $\left(7^{\prime}\right)$ to obtain $b(0)=0$, since $a \neq 1$. Now $\left(6^{\prime}\right)$ implies that $e(0)=$ 0 . Since we assumed that $w(0) \neq 0$, we have a contradiction and $w$ is not stable at 0 .

We now investigate what happens on even manifolds. So let $n=\operatorname{dim} M=$ $2 k$.

LemMA 4.4. Let $w$ be a 1-form on $M^{2 k}$ at $p$ such that $w(p)=0$. Then $w$ is not stable at $p$.

Proof. Generically, if $w(p)=0$, then $p$ is an isolated zero for $w$ and $(d w)^{k}(p) \neq 0$. Otherwise we would require more than $n$ functions to be simultaneously zero on a manifold of dimension $n$. Then $d w$ is symplectic near $p$ and there is a unique vector field $V$ such that $w=V\lrcorner d w$. Also $V$ has an isolated zero at $p$. As in Lemma 2.1, the eigenvalues of the linear point of $V$ at $p$ are invariants of $V$ under changes of coordinates. By perturbing $w$ by arbitrary closed forms, we can perturb $V$ arbitrarily amongst Hamiltonian vector fields. In this way we can change the eigenvalues of the linear part of $V$ by using a small perturbation of $w$. Thus $w$ is not stable at $p$.

We now describe Martinet's results in this case.

LEMMA $4.5\left[4\right.$, p. 153]. Let $w$ be the germ of a 1 -form on $M^{2 k}$ at $p$ with $w(p) \neq 0$ and $(d w)^{k}(p) \neq 0$; then there exist coordinates $x_{1}, y_{1}, \ldots, x_{k}, y_{k}$ on $M$ at $p$ such that

$$
w=\left(1+x_{1}\right) d y_{1}+x_{2} d y_{2}+\cdots+x_{k} d y_{k} .
$$

Lemma 4.6 $\left[4\right.$, p. 154]. Let $w$ be a 1 -form on $M^{2 k}$ at $p$ with $w(p) \neq 0$, $(d w)^{k}(p)=0$ (generically), and $w \wedge(d w)^{k-1}(p) \neq 0$. Then there exist coordinates $x_{1}, y_{1}, \ldots, x_{k}, y_{k}$ on $M^{2 k}$ at $p$ such that

$$
w=\left(1 \pm x_{1}^{2} / 2\right) d y_{1}+x_{2} d y_{2}+\cdots+x_{k} d y_{k} .
$$

Note. Let $X$ be the hypersurface defined by $(d w)^{k}=0$. Then off $X d w$ is symplectic and there is a unique vector field $V$ such that $w=V\lrcorner d w$. The 
sign of $x_{1}^{2} / 2$ is determined by whether the one parameter group of $V$ flows toward $(-)$ or away from $(+) X$.

Now let $w$ be the germ of a stable 1 -form on $M^{2 k}$ at $p$. Using Lemmas $4.4,4.5$, and 4.6 we may assume that
(A) $w(p) \neq 0$,
(B) $(d w)^{k}(p)=0$,
(C) $w \wedge(d w)^{k-1}(p)=0$.

Next we assume that

(D) $(d w)^{k-1}(p) \neq 0$.

The stability of $w$ implies that (B) holds transversely. Let $X^{2 k-1}=\{q \in M \mid$ $(d w)^{k}(q)=0$ \} and let $i: X \hookrightarrow M$ be the inclusion map. Transversality implies that $X$ is a hypersurface in $M$. We also assume

(E) $i^{*}(d w)$ has the maximum rank possible; that is $i^{*}(d w)^{k-1}(p) \neq 0$. Martinet calls a singularity of $d w$ at $p$ satisfying (B), (D), and (E) a $\Sigma_{2,0}$ singularity and proves the following: There exist coordinates $x_{1}, \ldots, x_{n}, y_{1}, \ldots, y_{n}$ on $M$ at $p$ such that $d w=x_{1} d x_{1} d y_{1}+d x_{2} d y_{2}+\cdots+d x_{k} d y_{k}$. See [4, p. 157].

So we may assume that $X=\left\{x_{1}=0\right\}$ and that $i^{*} w=x_{2} d y_{2}+\cdots+$ $x_{k} d y_{k}+d f$ where $f:(X, p) \rightarrow(R, 0)$. Now let

$$
T=\left\{q \in X \mid i^{*}\left(w \wedge(d w)^{k-1}\right)(q)=0\right\}
$$

and $j: T \hookrightarrow M$ be the inclusion map. In coordinates $T=\left\{\partial f / \partial y_{1}=0=x_{1}\right\}$. The stability of $w$ implies that the zeroes of $i^{*}\left(w \wedge(d w)^{k-1}\right)$ occur generically, so $T$ is a submanifold of codimension one in $X$. Moreover, $d\left(\partial f / \partial y_{1}\right)(p) \neq 0$. Assume

(F) $j^{*}(d w)^{k-1}(p) \neq 0$.

Now (F) is equivalent to $\operatorname{Ker} i^{*}(d w) \pitchfork T$ at $p$. Since $\operatorname{Ker} i^{*}(d w)=\left(\partial / \partial y_{1}\right)$ we have that $d\left(\partial f / \partial y_{1}\right)_{p}\left(\partial / \partial y_{1}\right) \neq 0$, or $\partial^{2} f(p) / \partial y_{2}^{2} \neq 0$. Note that (F) implies (E) when $X$ and $T$ are submanifolds.

Proposition 4.7. Let $w$ be the germ of a 1-form satisfying (A)-(F). Then $w$ is not stable at $p$.

Note. For $w$ to be stable it would have to satisfy these conditions generically (where applicable); so the discussion above holds.

LEMMA 4.8. Under the above hypotheses there exists a unique vector field $V$ on $X$ such that

(a) $V\lrcorner i^{*}(d w)=0$, and

(b) $V\left[\left(i^{*} w\right)(V)\right]= \pm 1$. 
Proof. As above we assume that $d w=x_{1} d x_{1} d y_{1}+d x_{2} d y_{2}+\cdots+$ $d x_{k} d y_{k}$. So Ker $i^{*} d w=\left(\partial / \partial y_{1}\right)$; if $V$ exists (a) implies that $V=a \partial / \partial y_{1}$ for some function $a$. Since $i^{*} w=x_{2} d y_{2}+\cdots+x_{n} d y_{n}+d f$ we have that

Letting $b=a^{2}$ yields

$$
V\left[\left(i^{*} w\right)(V)\right]=a \frac{\partial a}{\partial y_{1}} \frac{\partial f}{\partial y_{1}}+a^{2} \frac{\partial^{2} f}{\partial y_{1}^{2}} .
$$

$$
V\left[\left(i^{*} w\right)(V)\right]=\frac{1}{2} \frac{\partial b}{\partial y_{1}} \frac{\partial f}{\partial y_{1}}+b \frac{\partial^{2} f}{\partial y_{1}^{2}} .
$$

The lemma reduces to the following; does there exist a unique solution to the differential equation

$$
\frac{1}{2} \frac{\partial b}{\partial y_{1}} \frac{\partial f}{\partial y_{1}}+b \frac{\partial^{2} f}{\partial y_{1}^{2}}= \pm 1
$$

with $b(0)>0$ ? If so, let $a=\sqrt{ } b$. This depends on $f$, of course. Solving the corresponding homogeneous differential equation by separation of variables yields $b=K /\left(\partial f / \partial y_{1}\right)^{2}$ whe. $K=K\left(x_{2}, \ldots, x_{k}, y_{2}, \ldots, y_{k}\right)$. As we saw above, $\partial f(p) / \partial y_{1}=0$. So these solutions are undefined at $p=0$ unless $K \equiv 0$, and if a solution to (***) exists, it is unique.

We can now solve (***) by variation of parameters. Let $b=K /\left(\partial f / \partial y_{1}\right)^{2}$ where $K$ depends on $y_{1}$ as well. Then

$$
\frac{1}{2} \frac{\partial b}{\partial y_{1}} \frac{\partial f}{\partial y_{1}}+b \frac{\partial^{2} f}{\partial y_{1}^{2}}=\frac{1}{2} \frac{\partial K}{\partial y_{1}} / \frac{\partial f}{\partial y_{1}} .
$$

Define $\varphi\left(x_{2}, \ldots, y_{k}\right)$ implicitly by

$$
\left(\partial f / \partial y_{1}\right)\left(\varphi, x_{2}, \ldots, y_{n}\right)=0 \text { and } \varphi(0)=0 .
$$

This is possible since $\partial^{2} f(0) / \partial y_{1}^{2} \neq 0$. Then let

$$
K=2 \int_{\varphi\left(x_{2}, \ldots, y_{n}\right)}^{y_{1}} \frac{\partial f}{\partial y_{1}}\left(t, x_{2}, \ldots, y_{n}\right) d t .
$$

By construction $b=K /\left(\partial f / \partial y_{1}\right)^{2}$ is defined at $0\left(\right.$ since $\left.\partial^{2} f(p) / \partial y_{1}^{2} \neq 0\right)$ and solves the equation

$$
\frac{1}{2} \frac{\partial b}{\partial y_{1}} \frac{\partial f}{\partial y_{1}}+b \frac{\partial^{2} f}{\partial y_{1}^{2}}=1 \text {. }
$$

Now $b(0)=\left(\partial^{2} f(p) / \partial y_{1}^{2}\right)^{-1} \neq 0$. If $b(0)>0$, then $a=\sqrt{ } b$ solves $(* * *)$ and we are done. If not $c=-b$ solves the differential equation

$$
\frac{1}{2} \frac{\partial c}{\partial y_{1}} \frac{\partial f}{\partial y_{1}}+c \frac{\partial^{2} f}{\partial y_{1}^{2}}=-1
$$


with $c(0)=-b(0)>0$. So there is precisely one vector field $V$ on $X$ satisfying (a) and (b).

LEMMA 4.9. Under the hypotheses above, there exist vector fields $Y$ and $Z$ on $M$ such that

(1) $Z\lrcorner d w=0$ on $X$,

(2) $Y$ is an extension of $V$ on $X$,

(3) $Z d w(Z, Y)=1$ on $X$.

Moreover any two choices of $Z$ satisfying (1), (2), and (3) differ by some multiple of $V$ on $X$.

Proof. For existence, let $Y=a \partial / \partial y_{1}$ (as in the previous lemma) and $Z=$ $a^{-1 / 2} \partial / \partial x_{1}$ in the local coordinates chosen above for $M$. Note that $\partial a / \partial x_{1} \equiv 0$ and check that (1), (2), and (3) are satisfied.

Now let $Y$ and $Z$ be any vector fields satisfying (1), (2), and (3). Then

and

$$
Y=a \partial / \partial y_{1}+x_{1} Y_{1} \text { by (2) }
$$

$$
Z=b \partial / \partial x_{1}+c \partial / \partial y_{1}+x_{1} Z_{1} \text { by (1) }
$$

where $a, b$, and $c$ are independent of $x_{1}$ and $a$ is as above. To prove the moreover part of the lemma we need only show that $b$ is uniquely determined on $X$. For this, look at (3). In particular

$$
d w(Z, Y)=a b x_{1}+x_{1}^{2} l
$$

for some function $l$ and

$$
Z[(d w)(Z, Y)]=a b^{2} \text { on } X=\left\{x_{1}=0\right\} .
$$

So $b=a^{-1 / 2}$ on $X$. (Recall that $a(0)>0$ from the last lemma.)

LEMMA 4.10. Let $Z$ be a vector field satisfying the hypotheses of Lemma 4.9. Let $g: T \rightarrow \mathbf{R}$ be defined by $g=w(Z) \mid T$. Then $g$ is defined independently of the choice of $Z$. Moreover if $w^{\prime}=h^{*} w$ where $h:\left(M, p^{\prime}\right) \rightarrow$ $(M, p)$ is some diffeomorphism, then the corresponding $g^{\prime}: T^{\prime} \rightarrow \mathbf{R}$ is given by $g^{\prime}=g \circ h$.

Proof. On $T$ we have that

$$
\left.0=V\lrcorner i^{*}\left(w \wedge(d w)^{k-1}\right)=(V\lrcorner i^{*} w\right) \wedge i^{*}(d w)^{k-1} .
$$

So $V\lrcorner i^{*} w=0$ on $T$. Since $V$ is tangent to $X$ it follows that $\left.V\right\lrcorner w=0$ on

$T$. Apply the moreover part of Lemma 4.9 to see that $g$ is well defined on $T$.

The moreover part of this lemma is an easy functorial diagram chase.

Proof of Proposition 4.7. Let $j: T \hookrightarrow M$ be the inclusion map.

There are two possibilities; either 
(G1) $j^{*} w(p) \neq 0$, or

(G2) $j^{*} w(p)=0$.

We assume that (G1) holds first. Thus there is a nonzero vector field $U$ on $T$ such that $\left.j^{*} w=U\right\lrcorner j^{*} d w$. $U$ exists since $j^{*} d w$ is symplectic on $T$ by (F) and is nonzero by (G1). Let $\phi_{t}: T \rightarrow T$ be the infinitesimal generator for $U$. Define $\overline{\boldsymbol{g}}_{p}: \mathbf{R} \rightarrow \mathbf{R}$ by $\bar{g}_{p}(t)=g\left(\phi_{t}(p)\right)$. We claim that the germ of $\bar{g}_{p}$ at 0 is an invariant of the equivalence class of $w$; that is, if $w^{\prime}=h^{*} w$ for some diffeomorphism $h:\left(M, p^{\prime}\right) \rightarrow(M, p)$, then $\bar{g}_{p}=\bar{g}_{p^{\prime}}$. This is true since $U=h_{*} U^{\prime}$ and $g^{\prime}=g \circ h$ by Lemma 4.10 .

Let $J^{2 k}(\mathbf{R}, \mathbf{R})_{\circ}=(2 k)$-jets of mappings of $\mathbf{R} \rightarrow \mathbf{R}$ with source 0 . Then the form $w$ defines a map $\Psi_{w}: T \rightarrow J^{2 k}(\mathbf{R}, \mathbf{R})$ 。 defined by $q \longmapsto j^{2 k}\left(\bar{g}_{q}\right)(q)$. We claim that $w$ can be perturbed by an arbitrarily small perturbation to a 1 form $w^{\prime}$ so that the new $\Psi_{w^{\prime}}$ is transverse to any given submanifold $W$ of $J^{2 k}(R, R)_{0}$. Note that if the perturbation is small enough $\Psi_{w^{\prime}}$ is well defined. This will be enough to prove that any $w$ satisfying the hypotheses of this proposition and (G1) will not be stable at $p$. To see this, let $W=$ the point $j^{2 k}\left(\bar{g}_{p}\right)(p)$. Then the codimension of $W$ in $J^{2 k}(\mathbf{R}, \mathbf{R})_{\circ}$ is $2 k+1$. So $\Psi_{w^{\prime}} \Phi W$ implies that $\Psi_{w^{\prime}}\left(T^{\prime}\right) \cap W=\varnothing$ since $\operatorname{dim} T=2 k-2$. Hence at no point of $T^{\prime}$ is the (2k)-jet of $\bar{g}_{q}^{\prime}$ for $w^{\prime}$ equal to the (2k)-jet of $\bar{g}_{p}$ at 0 for $w$. So $w$ at $p$ is not equivalent to $w^{\prime}$ at any point $q$ in $T^{\prime}$. Since any diffeomorphism $h$ for which $h^{*} w^{\prime}=w$ must satisfy $h\left(T^{\prime}\right)=T$ we have that $w$ is not stable at $p$.

To prove the claim, let $l$ be a function on $M$ such that $l(X)=0$ and $(d l)(Z)$ $\equiv 1$ near $p$. Let $w_{k}=w+d(l k)$ for some function $k$. Define $V_{k}$ as in Lemma 4.8. But $V_{k}=V$ since $i^{*} w_{k}=i^{*} w$, and $X_{k}=X$ since $d w_{k}=d w$. Similarly we can let $Z_{k}=Z$; so $g_{k}=w_{k}(Z) \mid T$. Now $w_{k}=w+k d l$ on $X$; thus $g_{k}=g+k$. Since $j^{*} w_{k}=j^{*} w, U_{k}=U$. Therefore we can perturb $\bar{g}_{p}$ arbitrarily. Thus the map $T \times J^{k}(\mathbf{R}, \mathbf{R})_{\circ} \rightarrow J^{k}(\mathbf{R}, \mathbf{R})$ 。 given by $(q, k) \mapsto \Psi_{w+k}(q)$ is a submersion. By the fundamental transversality lemma (see [1, Remark, p. 54] there is a dense set of parameters $k$ near 0 for which $\Psi_{w+k}$ 瓜 a given submanifold $W$ of $J^{k}(\mathbf{R}, \mathbf{R})_{0}$. This proves the claim.

Next assume that $w$ satisfies the hypotheses of the lemma and (G2); i.e., $\left(j^{*} w\right)(p)=0$. Again the assumed stability of $w$ forces the zero of $j^{*} w$ to be generic and thus isolated. Similarly for any small perturbation $w^{\prime}$ of $w$, $\left(j^{\prime}\right)^{*}\left(w^{\prime}\right)\left(p^{\prime}\right)=0$ is isolated. Thus any diffeomorphism $h$ satisfying $h^{*} w^{\prime}=w$ at $p$ must satisfy $h(p)=p^{\prime}$. As above, the value $g(p)$ depends only on the equivalence class of $w$ at $p$; that is $g^{\prime}\left(p^{\prime}\right)=g(p)$ if $h^{*} w^{\prime}=w$ at $p$. Yet the above perturbations make it easy to change the value $g^{\prime}(p)$ while keeping the isolated zero of $j^{*} w^{\prime}$ at $p$. Thus, if $w$ satisfies (G2), it also is not stable. Q.E.D. 
THEOREM 4.11. On a 4-manifold $M^{4}$, any germ of a 1-form at $p$ satisfying (A), (B), and (C) is not stable.

Note. As a consequence of this theorem, we see that there are only two types of stable germs of 1-forms on 4-manifolds and they were given by Martinet as in Lemmas 4.5 and 4.6. We strongly suspect that this is the case on all even-dimensional manifolds.

Proof. Since $d w$ has six coordinate functions, generically they cannot all be zero [4, p. 107]. So if $w$ is stable, assumption (D) must hold. If (E) and (F) hold, then Proposition 4.7 states that $w$ is not stable at $p$. Next assume (E) fails; that is, $i^{*}(d w)(p)=0$. Since $i^{*}(d w)$ is a 2-form on the 3-manifold $X$, we may apply the reasoning in Lemma 2.1 to show that this cannot happen stably.

Finally assume that (E) holds while (F) fails. So $j^{*} d w(p)=0$. Let $W=$ $\left\{q \in T \mid j^{*} d w(q)=0\right\}$. Generically $W$ is a submanifold of dimension one. Let $S=\{q \in T \mid w \wedge d w(q)=0\}$. Generically $S$ is also a submanifold of $T$ of dimension one. We claim that if $w$ is stable, then $W \Phi S$ at $p$. Let $w^{\prime}=w+$ $d\left(x_{1} k\right)$ for some function $k$ with $k(0)=0$. Then $d w^{\prime}=d w$ and $i^{*} w^{\prime}=i^{*} w$, so $T^{\prime}$ $=T$. Also $W^{\prime}=W$. But $S^{\prime}$ is given by $w^{\prime} \wedge d w^{\prime}=0$, which we compute by noting that

$$
w^{\prime} \wedge d w^{\prime} \mid X=\left(w+k d x_{1}\right) d x_{2} d y_{2} .
$$

Thus we can change the coefficient of the term $d x_{1} d x_{2} d y_{2}$ arbitrarily on $T$. This is enough to change the direction of $S^{\prime}$ at $p$ and proves the claim. Note that now the point $p$ is distinguished by $W \cap S=\{p\}$.

Next we claim that $j^{*} w(p) \neq 0$. For $j^{*} w$ is a 1 -form on the 2-manifold $T$. Applying the reasoning in Lemma 2.1 again we see that $j^{*} w$ cannot equal zero at $p$ stably.

Since $j^{*} w(p) \neq 0, \operatorname{Ker} j^{*} w$ is a well-defined line field on $T$. We claim that if $w$ is stable, then $\operatorname{Ker} j^{*} w \Phi W$ at $p$ and $\operatorname{Ker} j^{*} w \Phi S$ at $p$. Using the perturbation above; namely, $w^{\prime}=w+d\left(x_{1} k\right)$, we see that $j^{*} w^{\prime}=j^{*} w$ while $S^{\prime}$ can be rotated arbitrarily from $S$. So we have that stability implies that $\operatorname{Ker} j^{*} w S$ at $p$. Suppose that $\operatorname{Ker} j^{*} w(p)=T_{p} W$. Stability implies that the line field $\operatorname{Ker} j^{*} w$ be tangent to $W$ at an isolated point. Thus $p$ is the unique point where $W$ meets $S$ and where $\operatorname{Ker} j^{*} w=T W$. Both of these properties are preserved under pull-back via a diffeomorphism. Use the same type of perturbation. As before $j^{*} w^{\prime}=j^{*} w$, so $W^{\prime}=W$. Yet if we assume that $k(0) \neq 0$, then $w^{\prime} \wedge d w^{\prime} \mid X$ is not zero at $p$, so $p$ is not in $S$. Now $S \cap W \neq\{p\}$ while $\operatorname{Ker} j^{*} w(p)=T_{p} W$. So stability also implies that $\operatorname{Ker} j^{*} w \Phi W$ at $p$.

We have the following situation: If $w$ is stable at $p$, then there exist onedimensional submanifolds $W$ and $S$ of $T$ with $W \cap S=\{p\}$ and a nonzero line field $\operatorname{Ker} j^{*} w$ transverse to both $W$ and $S$ at $p$. Let $\pi: T \rightarrow S$ be the projection 
map defined by $\operatorname{Ker} j^{*} w$. Then $\pi \mid W: W \rightarrow S$ is a germ of a diffeomorphism on $W$ at $p$. There also exist natural coordinates on both $W$ and $S$. Let $k: S \hookrightarrow M$ and $l: W \hookrightarrow M$ be the respective inclusion maps. Then $k^{*} w$ and $l^{*} w$ are both not zero at $p$ since $\operatorname{Ker} j^{*} w \boldsymbol{T} S$ and $W$ at $p$. So there exist functions $x$ on $W$ and $y$ on $S$ such that $x(p)=y(p)=0, d x=l^{*} w$, and $d y=k^{*} w$. Using these coordinates $\pi \mid W$ yields the germ of a diffeomorphism $g:(R, 0) \rightarrow(R, 0)$. This germ is an invariant of the equivalence class of $w$ as it is defined in a totally functional way. Since the perturbations described above give a way of rotating $S$ while fixing $W$ and the line field $\operatorname{Ker} j^{*} w$, we can change the diffeomorphism $g$ by small perturbations of $w$. So in this case, too, $w$ is not stable at $p$. Q.E.D.

\section{BIBLIOGRAPHY}

1. M. Golubitsky and V. Guillemin, Stable mappings and their singularities, Graduate Texts in Math., vol. 14, Springer-Verlag, New York, $1973 . \quad$ MR 49 \#6269.

2. M. Golubitsky and D. Tischler, The nonexistence of globally stable forms, Proc. Amer. Math. Soc. 58 (1976), 296-300.

3. C. Hsiung, On the stability of differential forms, Thesis, University of Illinois, Urbana, III., 1973.

4. J. Martinet, Sur les singularitées des formes différentielles, Ann. Inst. Fourier (Grenoble) 20 (1970), fasc. 1, 95-178.

5. D. Schaeffer, A regularity theorem for conservation laws, Advances in Math. 11 (1973), 368-386. MR 48 \#523.

6. G. Wasserman, Stability of unfoldings, Lecture Notes in Math., vol. 393, SpringerVerlag, New York, 1974.

7. E. C. Zeeman and D. J. A. Trotman, The classiflcation of elementary catastrophes of codimension < 5, Lecture Notes of Mathematics Institute, Univ. of Warwick, June 1974.

DEPARTMENT OF MATHEMATICS, QUEENS COLLEGE (CUNY), FLUSHING, NEW YORK 11367 